\title{
Diversity Combining Techniques to Mitigate Multipath Fading in Mobile Wireless Communications
}

\author{
Falade A. J. ${ }^{1}$, and O. T.Ibikunle ${ }^{1}$, and A.T. Ajiboye ${ }^{2}$ \\ 1. Department of Electrical and Electronics Engineering, Faculty of Engineering and Technology, University of \\ Ilorin, Nigeria. \\ 2. Engineering and Scientific Services Department, National Centre for Agricultural Mechanization, Ilorin.
}

\begin{abstract}
The work presents the investigation of GMSK and BPSK transmission schemes with both MRC and EGC diversity combining techniques over Rayleigh flat fading channels in a digital mobile wireless system. The System model for the received signal was developed under two propagation paths and simulated. Randomly generated symbols and Image data, were used as the Information signal. The results obtained showed that BPSK had a higher error probability values than the corresponding GMSK scheme. The results showed that due to GMSK high immunity to noise and other interfering signals, it provides a better performance than BPSK signalling scheme. Also, MRC was observed to perform better in terms of mitigation of the interference induced by the Rayleigh channel than EGC diversity combining technique. The quality of the images received with both signalling scheme were in agreement with the BER results.
\end{abstract}

Keywords: Multipath fading, Diversity combining, Rayleigh channel, Bit error rate

\section{Introduction}

The effectiveness of a communication system is a measure of the channel bandwidth and immunity to interference or noise. This ensures the maintenance of data integrity during transmission. When a signal is transmitted over a radio channel in a digital mobile wireless communication system, it is subjected to absorption, reflection, refraction and diffraction. Especially in the urban and suburban areas where cellular phones are often used, the communication environment changes quickly and thus introduces more complexities and uncertainties to the channel response. These multiple physical phenomena are responsible for most of the characteristic features of the received signal. The major impediments for signal detection at the physical layers are multipath fading and multiple access interference caused by co-channel users which are not orthogonal to the desired user (Constantinos and Howard, 2001).

At the receiver, these multipath waves with randomly distributed amplitudes and phases combine to give a resultant signal that fluctuates in time and space. Therefore, a receiver at one location may have a signal different from the signal at another location, only a short distance away. Signal fading arises from multiple transmission paths at the receiver with different phase shift, delay spread which is the time spread between the arrival of the first and last multipath signal seen by the receiver (Adeyemo and Abolade, 2011; Adeyemo and Teslim, 2010). The delay spread in a digital communication system often leads to inter-symbol interference (ISI).

In this work, the channel is assumed to be frequency non-selective flat fading, and ISI is not present. Random data were generated and modulated with each of the modulation schemes. The result is filtered using Gaussian low pass for GMSK to reduce the spectral spreading of the sidebands. Also, a rectangular pulse shaping filter is employed to reduce ISI induced in the system for BPSK to achieve suitable transmission through the wireless mobile channel. The bit error rate is the measure used in the performance evaluation.

\section{System model}

A single mobile to base station communication with one antenna over a frequency non-selective channel was considered first and other interfering signals are added together and modelled as additive white Gaussian noise. The received signal by the base station through only one path is given by (Adeyemo and Teslim, 2010) as:

$r(t)=h(t, T) S(t)+n(t)$

Where $h(t, T)$ is the time varying impulse response of the channel, $S(t)$ is the transmitted GMSK and BPSK signal and is equal to $S G B(t)$ and $n(t)$ is interfering signals modelled as AWGN

For GMSK scheme, the signal is represented by $m(t)=\sin \left(2 \pi f_{c} t\right) I(t)+\cos \left(2 \pi f_{c} t\right) Q(t)$ (2) where $f_{c}$ is the carrier frequency, $I(t)$ is the inphase baseband signal and $Q(t)$ is the quadrature baseband signal. The 
inphase and quadrature baseband signals are the cosine and sine of the output of the integrator from Gaussian LPF.

\section{Maximum ratio combining (mrc)}

The channel impulse response is the weight which when multiplied by each of the corresponding signal paths cancel the phase variations in the channel. Hence,

$h(t, T)=\alpha_{i}(t, T) \exp \left(j \theta_{i}(t, T)\right) \sigma\left(T-T_{i}(t)\right)$

Where $\alpha_{i}(t, T)$ is the amplitude of $\mathrm{i}^{\text {th }}$ multipath at $t$ and $T$, and $\sigma$ is the impulse function that determines the specific multipath component at time $t$ and delay $T$.

With two independent identically distributed paths, the received signal $S_{G, B}(t)$ is $S_{G, B}(t)=h_{0}^{*}(t, T) r_{0}(t)+h_{1}^{*}(t, T) r_{1}(t)$

Where $h_{0}{ }^{*}(t, T)$ and $h_{1}{ }^{*}(t, T)$ are the complex conjugate of the channel impulse responses of path 1 and 2 , and $r_{0}(t)$ and $r_{1}(t)$ are the received signals through paths 1 and 2 respectively.

\section{Equal gain combining (egc)}

In an EGC receiver, as with the previous case of MRC, both branch signals are multiplied by the same branch gain $(\mathrm{G})$ and the resulting signals are co-phased and summed. The resultant output signal is connected to the demodulator, therefore, equation (4) can be re-written as $S_{G, B}(t)=G_{0}(t, T) r_{0}(t)+G_{1}(t, T) r_{1}(t)$

Where $G_{0}(t, T)$ and $G_{1}(t, T)$ are the gain of each path 1 and 2 respectively.

\section{Mobile multipath channel parameters:}

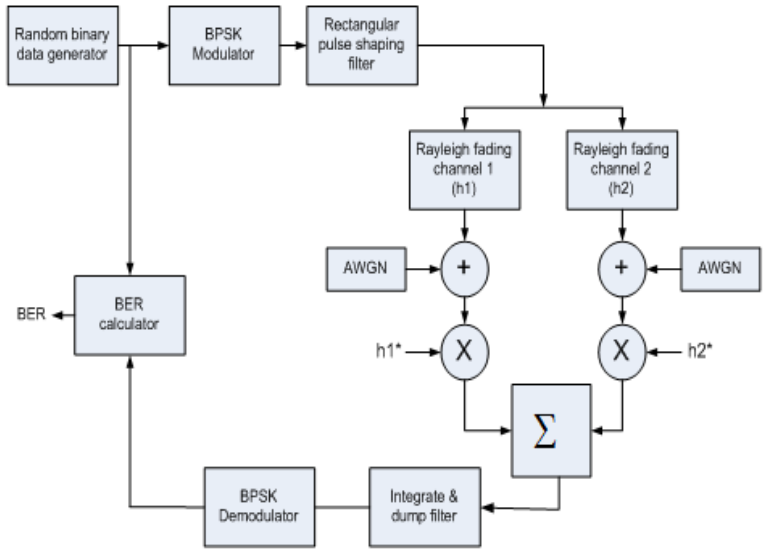

Figure 1: Simulation model for MRC $(\mathrm{L}=2)$ with BPSK signalling scheme

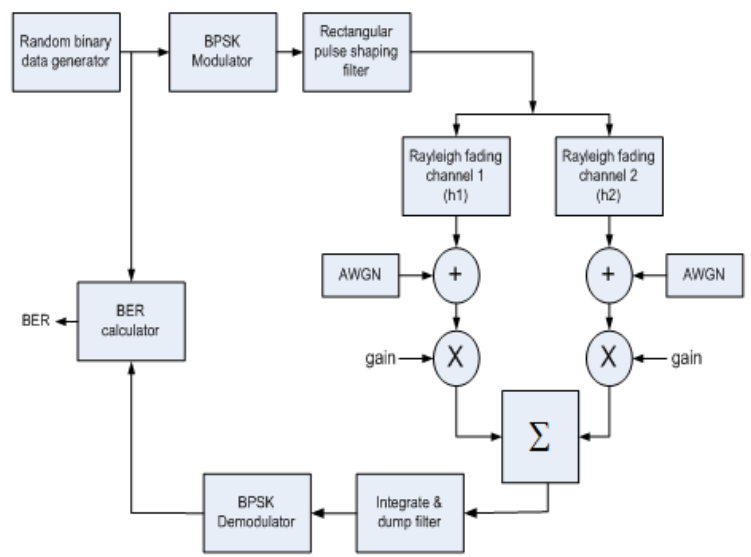

Figure 2: Simulation model for EGC $(\mathrm{L}=2)$ with BPSK signalling scheme 


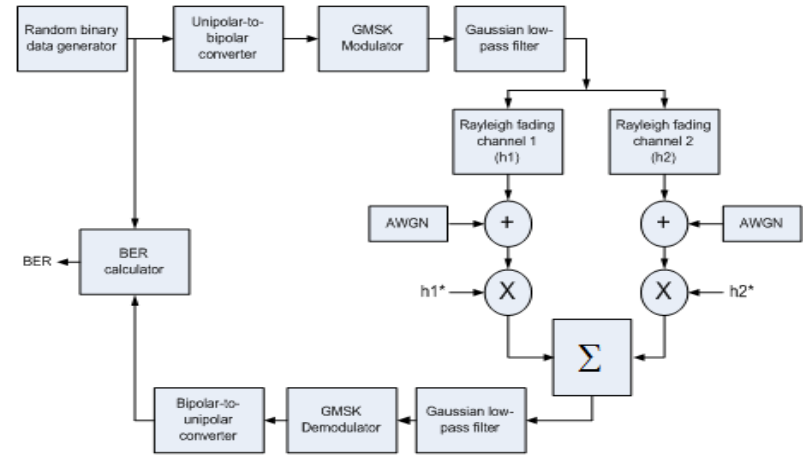

Figure 3: Simulation model for MRC $(\mathrm{L}=2)$ with GMSK signalling scheme

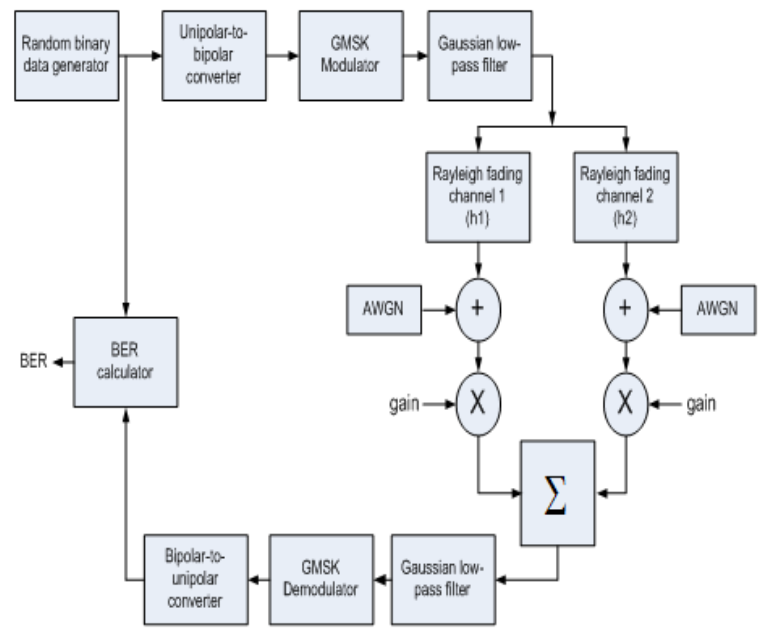

Figure 4: Simulation model for EGC $(L=2)$ with GMSK signalling scheme

To compare the performance of the BPSK with the GMSK signalling schemes, the system model simulation is given in figures 1 to 4 for BPSK with 2-path EGC/MRC, and GMSK with 2-path EGC/MRC.

\section{Data Acquisition}

Data sources for the simulation are those generated randomly and the image data of University of Ilorin Senate Building (UISB). Random data is used to determine the actual error bits while image signal complements the output of the results. The parameters and system configuration used in the simulation were chosen from the standard values. Doppler spread was computed to be $25 \mathrm{H}_{\mathrm{Z}}$ and the coherence time is $4 \times 10^{-2} \mathrm{sec}$. The multipath delay spread of $2.0 \times 10^{-7} \mathrm{sec}$ gives $5 \mathrm{mhz}$. This is larger than the assumed bandwidth of $200 \mathrm{H}_{\mathrm{Z}}$ indicating frequency non-selective /flat fading channel.

\section{Results And Discussion}

The results obtained from simulation of both random data and UISB image over a Rayleigh frequency non-selective/flat fading channel for GMSK and BPSK signalling schemes using both MRC and EGC at the receiver are shown in figures 5 to 11 . Figures 5 and 6 , are the BER performance of BPSK signalling scheme for both MRC and EGC respectively. 


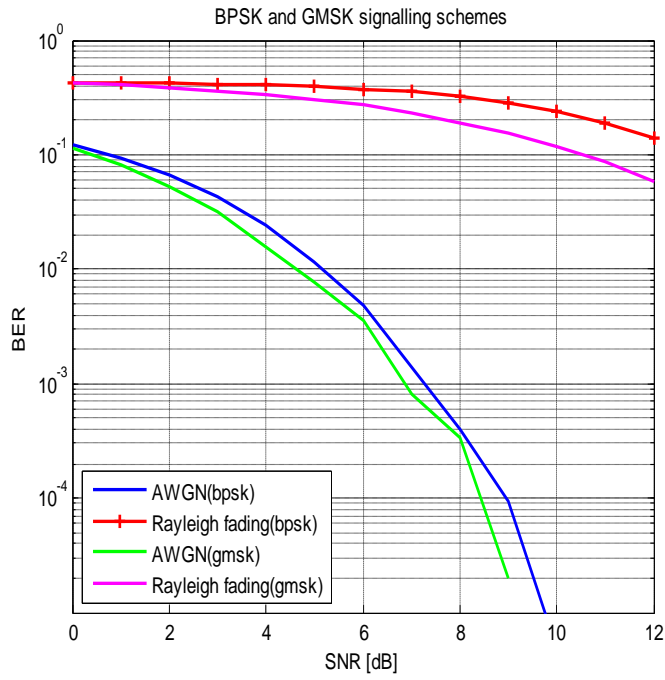

Fig. 5 Bit error rate of BPSK and GMSK modulation schemes over flat Rayleigh fading channel and AWGN channel.

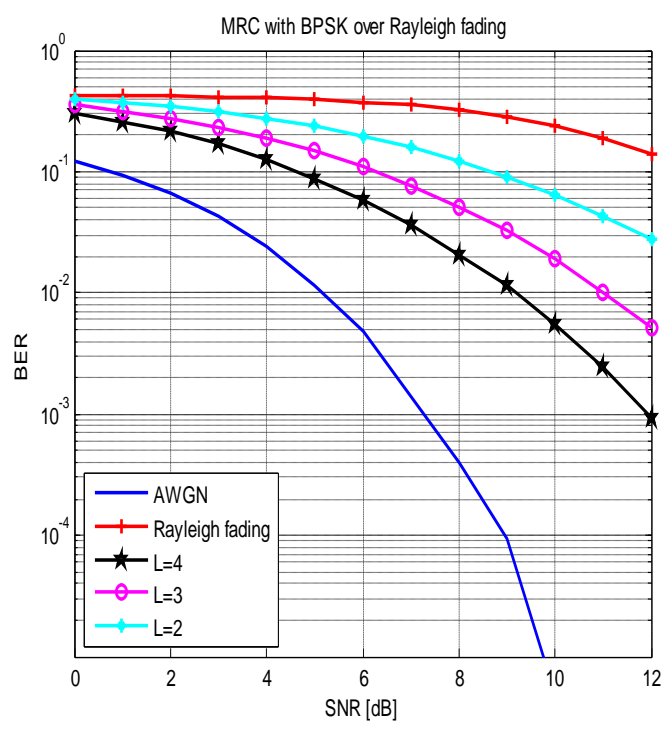

Fig. 6 Bit error rate comparison for varying paths Maximal Ratio Combining (MRC) using BPSK modulation scheme over Rayleigh channel.

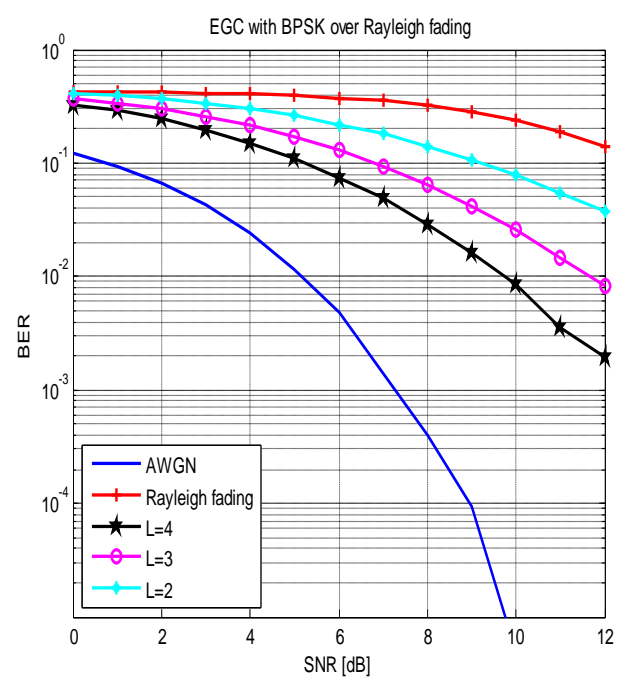

Fig. 7 Bit error rate comparison for varying paths of Equal Gain Combining (EGC) using BPSK modulation scheme over Rayleigh channel. 
The varying paths are assumed to be independent identically distributed. For 100000 bits of BPSK signals transmitted at SNR of 6db in Rayleigh flat fading channel, 37640 bits were changed by the channel, indicating number of bit errors. But with 2-paths, 3-paths and 4-paths MRC at the receiver, the high erroneous bits were reduced to 19780 bits, 10990 bits, and 5790 bits respectively. With 2-path, 3-paths, and 4-paths EGC at the receiver, the high erroneous bits rate were further reduced to 21980 bits, 12900 bits, and 7430 bits respectively. The results showed the effect of delay and doppler spread which resulted in a high erroneous bit when no diversity combiner was employed. The reduction in bit errors was as a result of varying paths in MRC and EGC diversity combiner separately employed at the receiver.

Figures 7 and 8, show the profile of BER performance of GMSK signalling scheme with the MRC and EGC at the receivers over a Rayleigh channel respectively. For a total of 100000 bits of GMSK signals transmitted at SNR of 6db in Rayleigh flat fading channel, 26930 bits were changed by the channel indicating number of bit errors. But with the introduction of 2-paths, 3-paths and 4-paths MRC at the receiver, the high erroneous bits were reduced to 13260 bits, 7570 bits, and 4320 bits respectively.

Also with 2-path, 3-paths, and 4-paths EGC at the receiver, the high erroneous bit are reduced to 16560 bits, 9870 bits, and 6340 bits respectively. The high erroneous bit were due to the effects of delay and doppler spread without diversity combiner.. The reduction in bit errors was as a result of varying paths of MRC and EGC diversity combiner.

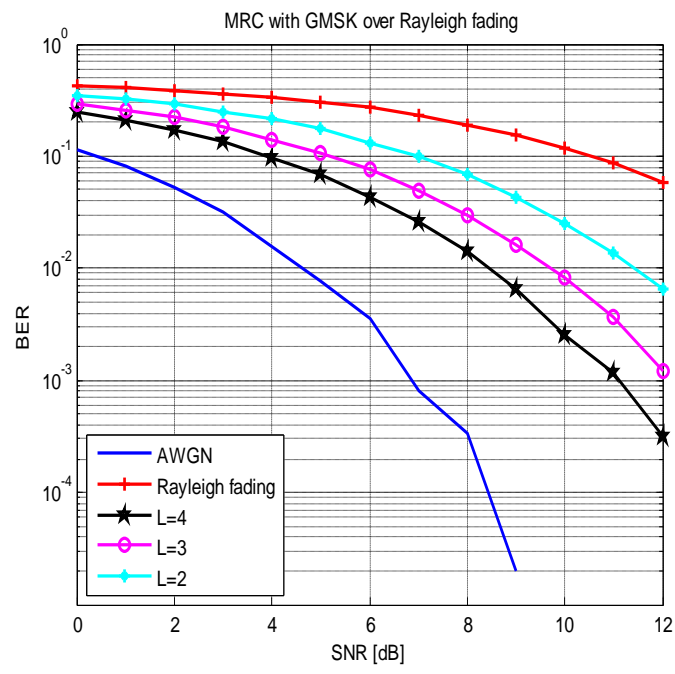

Fig. 8 Bit error rate comparison for varying paths of Maximal Ratio Combining (MRC) usingGMSK modulation scheme

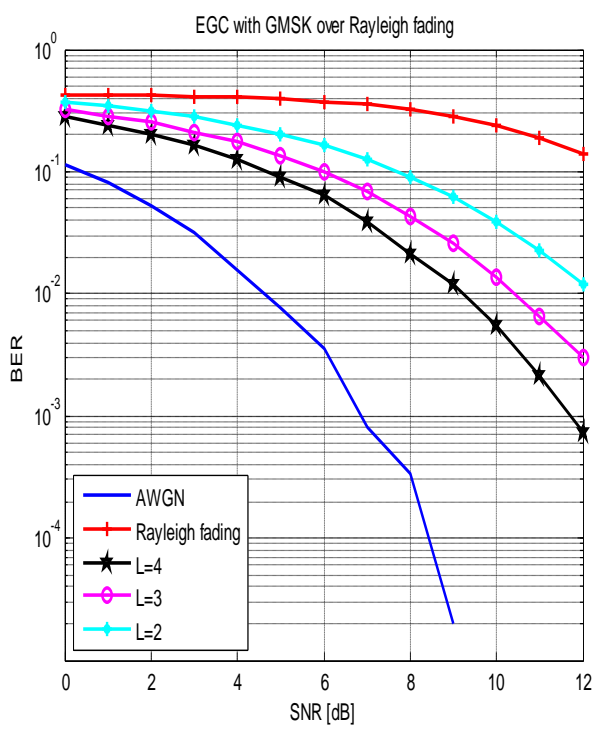

Fig. 9 Bit error rate comparison for varying paths of Equal Gain Combining (EGC) using GMSK modulation scheme. 
Figure 10 shows the application of diversity combining techniques to image data at SNR of $12 \mathrm{db}$ using BPSK. The image transmitted through an AWGN channel produced a close result compared with the original transmitted signal. When subjected to a Rayleigh fading channel at SNR of 12db, there was a great distortion in the original message. When 2-path of MRC/EGC was employed at the receiver, a better image output than in a flat Rayleigh fading channel was obtained. The employment of 3-path MRC/EGC and 4-path MRC/EGC produced a better image at the output in order of higher paths. The image quality output was improved for the two diversity combiner but MRC provided a better image than EGC. Figure 11 shows the application of diversity combining techniques to image data at SNR of $12 \mathrm{db}$ using GMSK. An image was transmitted through an AWGN channel which produced a close result to the original transmitted signal, but when subjected to a Rayleigh fading channel at SNR of $12 \mathrm{db}$, there was a great distortion in the original message. When a 2-path MRC/EGC was employed at the receiver, it gives a better image output than in a flat Rayleigh fading channel, but the employment of 3-path MRC/EGC provides a better result than a 2-path MRC/EGC diversity, while 4path MRC/EGC has the best image. The image output further supports the results from the profile, when comparing the image of MRC and EGC. For the same L-path diversity, though both EGC and MRC provides an improvement in the output, but MRC has a better image than EGC.

\section{Conclusion}

The system model for the received signal at a mobile speed of $30 \mathrm{~km} / \mathrm{hr}$ has been developed and simulated. The BER for each system was evaluated and for varying paths MRC and EGC diversity combiner, the performance is dependent on number of diversity branches. BPSK signalling schemes with both diversity combiner techniques separately employed at the receiver with MRC presented a clearer image than EGC diversity combiner.

\begin{tabular}{|c|c|c|}
\hline Original Image & AWGN effect & Rayleigh fading effect \\
\hline 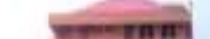 & 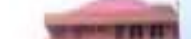 & 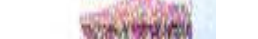 \\
\hline Fe: Tyes & 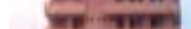 & gavows. \\
\hline mar-gen & mar-vers: & whed tow \\
\hline 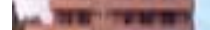 & 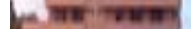 & tew \\
\hline ment Tren & 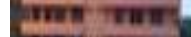 & 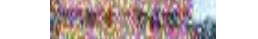 \\
\hline 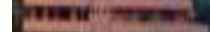 & 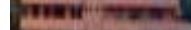 & 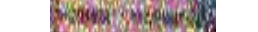 \\
\hline ment; wers & men:; wern & why swesgog \\
\hline & & \\
\hline
\end{tabular}

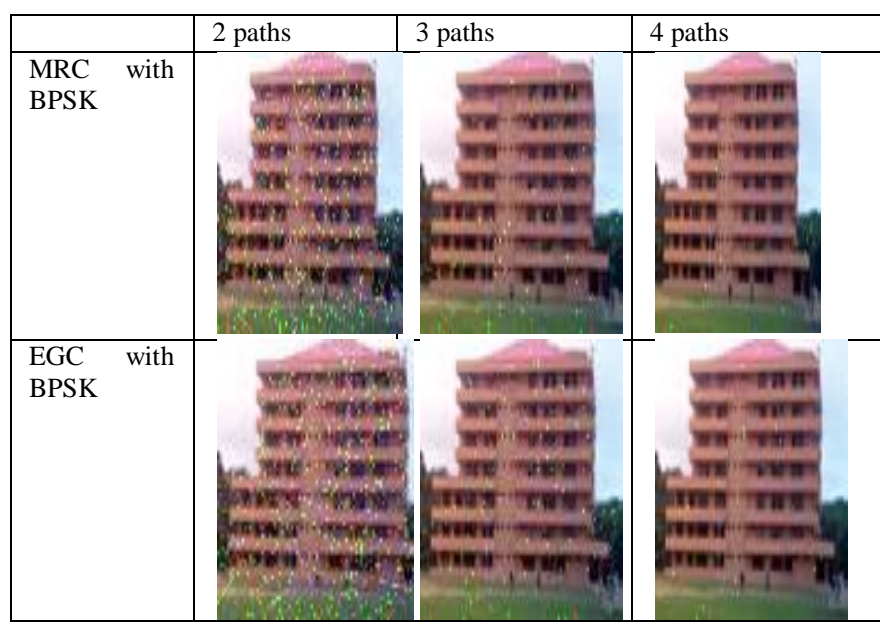

Fig. 10 Application of diversity combining techniques to image data at SNR of 12 db using BPSK

\begin{tabular}{|c|c|c|}
\hline Original Image & AWGN effect & Rayleigh fading effect \\
\hline - & 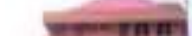 & 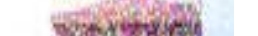 \\
\hline Sox & (x) & 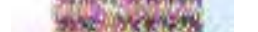 \\
\hline 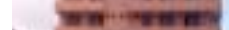 & 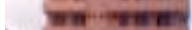 & Wistosed \\
\hline 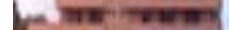 & 6 का & net. \\
\hline 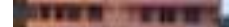 & mertr- TuE & 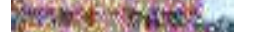 \\
\hline 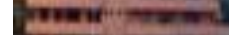 & 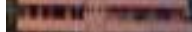 & 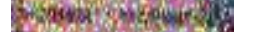 \\
\hline 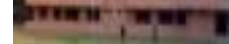 & 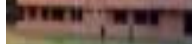 & 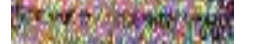 \\
\hline & & \\
\hline
\end{tabular}




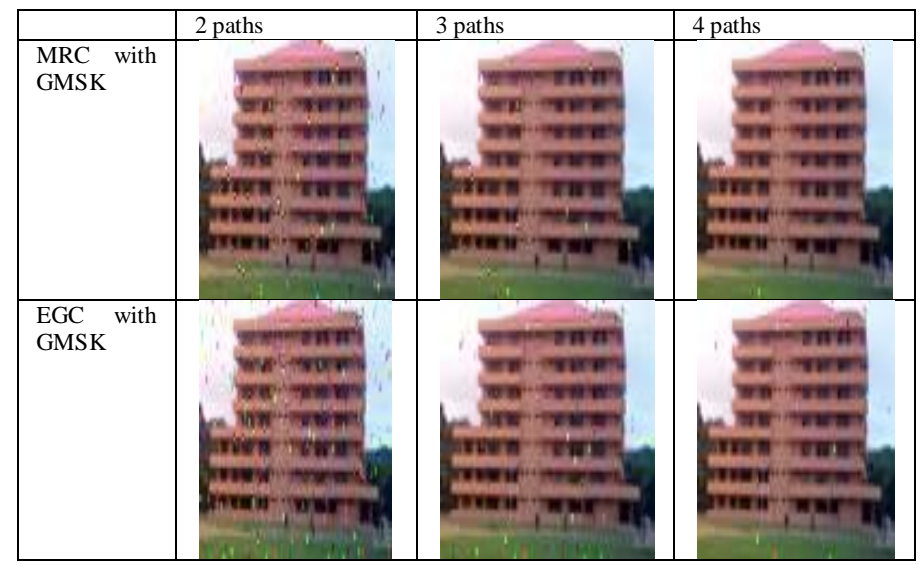

Figure 11 Application of diversity combining techniques to image data at SNR of $12 \mathrm{db}$ using GMSK.

The simulation results also showed that GMSK signalling scheme performed better than BPSK signalling when both were subjected to the same Rayleigh frequency non-selective fading channel, due to lower error bits received when transmitting the same number of bits. This shows GMSK is more immune to noise. In GMSK signalling schemes for image transmission with EGC and MRC combiner at the receiver, the result showed that though both diversity combiner techniques mitigated the effect of fading, but MRC presented a better image at the output than EGC diversity combining.

From these results, when comparing GMSK signalling scheme performance to BPSK signalling for image transmission using both MRC and EGC at the receiver, it was observed that GMSK signalling has a better performance in terms of a lower BER than BPSK signalling scheme. This is evident from the UISB image modulated by these signalling schemes. Therefore, the results showed that GMSK signalling scheme is better than BPSK when employing MRC diversity combining technique at the receiver with a Rayleigh frequency nonselective fading channel.

\section{References}

[1]. Adeyemo, Z. K., and Abolade, R. O., (2011) Comparative Analysis of GMSK and BDPSK signalling scheme with MRC over a Rayleigh fading environment. Advanced Materials Research Vol. 367 (2012) Pp 205-214, Trans Tech Publications, Switzerland.

[2]. Adeyemo, Z. K. And Teslim I. R., (2010) Bit error rate analysis for wireless links using Adaptive Combining Diversity. Journal of Theoretical and Applied Information Technology, Vol20, No 1, Pp 58-60.

[3]. Adeyemo, Z. K. And Teslim I. R., (2009) Performance of Binary phase shift keying (BPSK) and Quaternary phase shift keying (QSPK) Signalling schemes in Fast and frequency Non-selective Rayleigh fading channel with Decision Feedback Equalizer (DFE) at the Receiver. Advanced Materials Research, Trans Tech Publications, Switzerland, Vols.62-64, p.99-104.

[4]. Akbar, M. S., and Behnaam, A.. (1997), Joint multipath Doppler Diversity in Mobile Wireless Communications. Department of Electrical and Computer Engineering, University of Wisconsin-Madison Madison, WI 53706. IEEE Transactions On Communications, Vol. 47, No. 1, January 1999, pp123-132.

[5]. Aun, A. T., and Feng, Z. (2009) Performance Analysis on Modulation Techniques of W-CDMA in Multipath Fading Channel. Blekinge Institute of Technology School of Engineering, Department of applied signal processing. Available online at: www.slideshare.net/ Accessed on Feb. 20, 2013.

[6]. Constantinos and Howard, (2001) A linear time multiuser detector using antenna array processing for wireless CDMA capacity improvement. IEEE Journal on Selected Areas In Communications, Vol. 19, No. 2, February 2001

[7]. Deepmala, S. P., and Ravi, M., (2012), Channel Estimation in Multipath fading Environment using Combined Equalizer and Diversity Techniques. International Journal of Scientific \& Engineering Research Volume3, Issue 1, January 2012, ISSN 22295518.

[8]. Leon W. Couch, II, (2000) Digital and Analogue communication system. Prentice Hall $8^{\text {th }}$ edition.

[9]. Rappaport, T.S., (1996) Wireless Communication Principles and Practice, Prentice Hall, New Jersey.

[10]. Shankar, K. R., (2002) Bit error rate performance analysis of Zero Forcing (ZF) Equalizer, Maximum Likelihood (ML) Equalizer, Minimum mean squared zero (MMSE) Equalizers, for MIMO wireless communication Receiver. European Journal of Scientific Research ISSN 1450-216X Vol.59 No.4 (2011), pp. 522-53.2

[11]. Siavesh M. A., (1998) Simple Transmit diversity technique for wireless Communication, IEEE Journal on Selected Areas in Communication VOL 16: 8, pp 1451-58.

[12]. Zhiwei, Z. (2000) Digital communication via multipath fading channel. Xidian Univ. Press, 2000. 